care settings and populations. Bloodstream infections and resultant complications are common among hemodialysis patients; treating suspected infections and sepsis early is essential to quality care.

Notable strengths of the Hahn et al study include the examination of details associated with antibiotic starts on a larger scale than previous studies, including 1 year of data from $\sim 50$ dialysis facilities across Philadelphia County. The authors additionally demonstrated that collection of blood cultures prior to initiating empiric treatment for suspected bloodstream infections may be an area for improved antibiotic prescribing - a finding consistent with previous studies in this setting. ${ }^{4,5,7}$ Collection of blood cultures prior to initiating antibiotics for suspected bloodstream infections is integral to ensuring appropriate antibiotic selection and has the potential of improving infection cure rates. ${ }^{2,7}$

Antibiotic stewardship involves strategies to promote the optimal use of antibiotics. ${ }^{7}$ In the hemodialysis patient population, optimal use includes administration of empiric antibiotics to treat documented and suspected infections early and tailoring antibiotics based on culture results. Although routinely collected metrics for antibiotic use should be easily obtainable, oversimplified measures of appropriateness can potentially undermine the goals of stewardship and lead to misdirection of scarce resources. Expanding upon information captured through NHSN and validating new applications of the data might help to advance the science surrounding stewardship in outpatient dialysis settings. Additional, promising areas for intervention in this setting include standardization of blood culturing practices, improved communication among providers and across care transitions, and strengthened infection prevention programs and implementation. ${ }^{7}$
Acknowledgments. The findings and conclusions in this report are those of the authors and do not necessarily represent the official position of the Centers for Disease Control and Prevention.

Financial support. No financial support was provided relevant to this article.

Conflicts of interest. All authors report no conflicts of interest relevant to this article.

\section{References}

1. Hahn PD, Figgatt M, Peritz T, Coffin SE. Inappropriate intravenous antimicrobial starts: An antimicrobial stewardship metric for hemodialysis facilities. Infect Control Hosp Epidemiol 2019;40:1178-1180.

2. Standardization of blood culture collection for patients receiving in-center hemodialysis. Nephrologists Transforming Dialysis Safety website. https://www.asn-online.org/g/blast/files/

NTDS_Blood_Culture_Collection_Standardization_combined_01.16.2020. pdf. Published January 2020. Accessed February 8, 2021.

3. Dialysis event surveillance protocol. Centers for Disease Control and Prevention website. https://www.cdc.gov/nhsn/pdfs/pscmanual/8pscdialysis eventcurrent.pdf. Published February 2018. Accessed February 8, 2021.

4. Snyder GM, Patel PR, Kallen AJ, Strom JA, Tucker JK, D’Agata EM. Antimicrobial use in outpatient hemodialysis units. Infect Control Hosp Epidemiol 2013;34:349-357.

5. Zvonar R, Natarajan S, Edwards C, Roth V. Assessment of vancomycin use in chronic haemodialysis patients: room for improvement. Nephrol Dial Transplant 2008;23:3690-3695.

6. Green K, Schulman G, Haas DW, Schaffner W, D’Agata, EM. vancomycin prescribing practices in hospitalized chronic hemodialysis patients. Am J Kidney Dis 2000;35:64-68.

7. Apata IW, Kabbani S, Neu AM, et al. Opportunities to improve antibiotic prescribing in outpatient hemodialysis facilities. Am J Kidney Dis 2020. doi: 10.1053/j.ajkd.2020.08.011.

\title{
Time to remind us that absence of evidence is not evidence of absence during the coronavirus disease 2019 (COVID-19) pandemic
}

\author{
Chenyu Sun MD, MSc ${ }^{1}$ (1), Ce Cheng $\mathrm{DO}^{2}$ (1), Mubashir Ayaz Ahmed MD ${ }^{1}$ (1) and Qin Zhou $\mathrm{PhD}^{3}$ (1) \\ ${ }^{1}$ AMITA Health Saint Joseph Hospital Chicago, Chicago, Illinois, ${ }^{2}$ The University of Arizona College of Medicine at South Campus, Tucson, Arizona and ${ }^{3}$ Radiation \\ Oncology, Mayo Clinic, Rochester, Minnesota
}

To the Editor-During the current coronavirus disease 2019 (COVID-19) pandemic, guidelines issued by various agencies, including the US Centers for Disease Control and Prevention (CDC), have been conflicting on the issue of respiratory protection with a face mask or a respirator. ${ }^{1}$ The CDC has not officially announced the protective effects of masks for the wearers until recently, ${ }^{2}$ and even now, its web pages still show that surgical mask "is not considered respiratory protection." 3

\footnotetext{
Author for correspondence: Chenyu Sun, E-mail: drsunchenyu@yeah.net

Cite this article: Sun C, et al. (2022). Time to remind us that absence of evidence is not evidence of absence during the coronavirus disease 2019 (COVID-19) pandemic. Infection Control \& Hospital Epidemiology, 43: 675-676, https://doi.org/10.1017/ice.2021.47
}

Earlier this year, research on the protective effects of masks was limited, indicating lack of sufficient evidence to support the protective effects of masks to severe acute respiratory syndrome coronavirus 2 (SAS-CoV-2). However, the lack of evidence that masks have protective effects to respiratory viral infections is not equivalent to evidence that masks lack protective effects. It would be prudent to refrain from premature conclusions without further comprehensive studies. As Mark Twain said, "It ain't what you don't know that gets you into trouble. It's what you know for sure that just ain't so." At this time, we remind ourselves that the absence of evidence is not evidence of absence. ${ }^{4}$ Interestingly, while evidence of mask use against other viruses has not been strong enough for the CDC to suggest the protective effects of mask wearing, ${ }^{5}$ remdesivir was approved for emergency or experimental use, with only limited evidence, as a therapeutic candidate due to its ability to inhibit SARS-CoV-2 in vitro and against other 
coronaviruses. ${ }^{6}$ When it comes to wearing masks, a simple nonpharmaceutical intervention method with minimal side effects, how did the lack of evidence lead to recommendations against wearing them among the general public at the beginning of the pandemic? And even later, the CDC only recommended wearing masks to prevent asymptotic carriers and presymptomatic patients from spreading the virus. ${ }^{7}$

Again, we remind ourselves, when issues of public health are concerned, we must question whether the absence of evidence is a valid justification for inaction. ${ }^{8}$ Statements about the absence of evidence are common, such as protective effects of masks for the general public at the beginning of current COVID-19 pandemic. However, can we be comfortable that the absence of solid and clear evidence is equivalent to the position that masks provide no protective effects or only negligible effects? For this global threat, it is better to be safe than sorry, and we should take every possible reasonable intervention.

Financial support. No financial support was provided relevant to this article. Conflicts of interest. All authors report no conflicts of interest relevant to this article.

\section{References}

1. MacIntyre CR, Wang Q. Physical distancing, face masks, and eye protection for prevention of COVID-19. Lancet 2020;395:1950-1951.

2. Scientific brief: community use of cloth masks to control the spread of SARSCoV-2. Centers for Disease Control and Prevention website. https://www. cdc.gov/coronavirus/2019-ncov/more/masking-science-sars-cov2.html. Published 2020. Accessed February 3, 2021.

3. Understanding the difference. Centers for Disease Control and Prevention website. https://www.cdc.gov/niosh/npptl/pdfs/UnderstandDifference Infographic-508.pdf. Published 2020. Accessed February 3, 2021.

4. Alderson P. Absence of evidence is not evidence of absence. BMJ 2004;328:476-477.

5. Liang M, Gao L, Cheng C, et al. Efficacy of face mask in preventing respiratory virus transmission: A systematic review and meta-analysis. Travel Med Infect Dis 2020;36:101751.

6. Beigel JH, Tomashek KM, Dodd LE, et al. Remdesivir for the treatment of COVID-19- final report. N Engl J Med 2020. doi: 10.1056/NEJMoa2007764.

7. Federal Register notice: wearing of face masks while on conveyances and at transportation hubs. Centers for Disease Control and Prevention website. https://www.cdc.gov/quarantine/masks/mask-travel-guidance. html. Published 2020. Accessed February 3, 2021.

8. Altman DG, Bland JM. Absence of evidence is not evidence of absence. BMJ 1995;311:485.

\title{
Indirect transmission of severe acute respiratory syndrome coronavirus virus 2 (SARS-CoV-2): What do we know and what do we not know?
}

\author{
M. Khalid Ijaz DVM, MSc(Hons), PhD, FRSPH ${ }^{1,2}$, Raymond W. Nims PhD $^{3}$ (1) and Julie McKinney PhD ${ }^{1}$ \\ ${ }^{1}$ Global Research and Development for Lysol and Dettol, Reckitt Benckiser, LLC, Montvale, New Jersey, ${ }^{2}$ Department of Biology, Medgar Evers College, City \\ University of New York (CUNY), Brooklyn, New York and ${ }^{3}$ RMC Pharmaceutical Solutions, Longmont, Colorado
}

To the Editor-We wish to point out that 12 months into the coronavirus disease 2019 (COVID-19) pandemic, with >111 million reported cases and $>2.4$ million deaths, many knowledge gaps still need to be resolved empirically to fully appreciate the risks associated with high-touch environmental surface (HITES) contamination. It has been argued that indirect transmission through contaminated HITES is an unlikely route of transmission for severe acute respiratory syndrome coronavirus virus 2 (SARS-CoV-2) (eg, Goldman ${ }^{1}$ and Meyerowitz et $\mathrm{al}^{2}$ ) Why? Is this position based on data, and if so what data?

The World Health Organization ${ }^{3}$ rightly has made the point that it is difficult to separate potential direct and indirect exposure in establishing transmission relevancy. The safest approach is, therefore, to avoid discounting the possibility of indirect transmission until proper studies have been performed to support this view. ${ }^{4,5}$ Specifically, if we are to rule out indirect transmission as a likely route, we should do so based on adequate supporting data. What data do we need? The scenario in Figure 1 illustrates the primary knowledge gaps.

\footnotetext{
Author for correspondence: M. Khalid Ijaz, E-mail: Khalid.Ijaz@rb.com

Cite this article: Ijaz MK, Nims RW, and McKinney J. (2022). Indirect transmission of severe acute respiratory syndrome coronavirus virus 2 (SARS-CoV-2): What do we know and what do we not know?. Infection Control \& Hospital Epidemiology, 43: 676-678, https://doi.org/10.1017/ice.2021.57
}

What does our current knowledge tell us about the risk of acquiring infectious SARS-CoV-2 during the scenario illustrated in Figure 1? Unfortunately, the extent of deposition of infectious SARS-CoV-2 (not viral RNA determined by PCR assay!) onto the visitor's hand when he coughs, has not been empirically determined. On the basis of the accepted respiratory droplets or aerosol transmission route, one must assume that a significant burden of infectious virus would be discharged onto the hand by such a cough. If so, why haven't the data been generated to support this? We do know that SARS-CoV-2 can survive on skin for hours (half-life of 3-5 hours at room temperature). ${ }^{6,7}$ Assuming that the SARS-CoV-2-infected visitor leaves the office within the hour, the virus deposited on his hand while coughing should remain infectious until he reaches for the door knob. Here we run into another knowledge gap, for we have no empirical data to help us assess the quantity of infectious SARS-CoV-2 that might be transferred from the visitor's hand to the door knob. Once on the steel door knob, we do, however, have empirical data to help us predict how long infectious SARS-CoV-2 can remain there. For instance, half-life data on SARS-CoV-2 survival on experimentally contaminated prototypic HITES at room temperature exist from several investigators. Reaching for the contaminated door knob as you leave the 